\title{
IS ACQUISITION OF METHICILLIN-RESISTANT STAPHYLOCOCCUS AUREUS - AN OCCUPATIONAL HAZARD FOR MEDICAL STUDENTS IN INDIA?
}

\author{
SHREYAS K, RADHAKRISHNA M*, ASHWINI HEGDE, POOJA RAO \\ Department of Microbiology, Kasturba Medical College, Manipal University, Mangalore, Karnataka, India. \\ Email: manipuraradhakrishna@yahoo.com
}

Received: 20 June 2017, Revised and Accepted: 19 August 2017

\section{ABSTRACT}

Objective: The incidence of community-acquired and hospital-acquired Staphylococcus aureus infections has been rising with increasing emergence of drug-resistant strains called methicillin-resistant $S$. aureus (MRSA). The main objective of this study was to determine the prevalence of carriage rate of MRSA among the interns of tertiary care teaching hospital in Mangalore.

Methods: A total of 150 interns were participated, thereby swabs from both anterior nares were collected and processed for $S$. aureus. The isolated strains of $S$. aureus were screened for methicillin susceptibility by modified Kirby-Bauer method using cefoxitin (30 $\mu$ g) disc. Further, antibiotic susceptibility testing for all isolates of $S$. aureus was also done against selected appropriate antibiotics. Vancomycin susceptibility testing for MRSA was done by E test.

Results: The number of strains of $S$. aureus isolated from our 150 participants was 71 with a percentage rate of 47.3 . Of the 71 isolates of $S$. aureus, one $(1.4 \%)$ was MRSA. The overall MRSA carriage rate was $0.66 \%$. The $S$. aureus and MRSA carriage rates recorded in this study were at par and significantly lower, respectively, when compared with other reported studies. It was observed that risk factors such as the use of antibiotics in the past 6 months and smoking were found to be statistically significant in nasal carriage status of $S$. aureus $(<0.05)$. The only one isolate of MRSA was found to be sensitive to vancomycin, linezolid, and teicoplanin.

Conclusion: It was concluded that the acquisition of $S$. aureus and MRSA would not be an occupational hazard for medical students of developing countries like India.

Keywords: Staphylococcus aureus, Methicillin-resistant Staphylococcus aureus, Nasal carriage, Medical interns.

(C) 2017 The Authors. Published by Innovare Academic Sciences Pvt Ltd. This is an open access article under the CC BY license (http://creativecommons. org/licenses/by/4. 0/) DOI: http://dx.doi.org/10.22159/ajpcr.2017.v10i12.20865

\section{INTRODUCTION}

Staphylococcus aureus is considered as one of the most frequently occurring community and hospital-acquired pathogens. Nasal carriage of $S$. aureus represents a major risk factor for subsequent invasive infections and interpatient transmission of strains [1]. Infections caused by $S$. aureus are found in various anatomic sites including surgical sites and the skin, the lower respiratory tract, the bloodstream, vascular catheters, and the urinary tract. Although primary S. aureus infections are not common, a great deal of the virulence from this organism occurs through cross-infection by spread from patient to patient in hospitals and other institutional settings [2]. The incidence of community-acquired and hospital-acquired $S$. aureus infections has been rising with increasing emergence of drug-resistant strains called methicillin-resistant S. aureus (MRSA) [3]. Both MRSA and methicillinsensitive $S$. aureus (MSSA) infections have significant morbidity and mortality rates ranging from $8 \%$ to $49 \%$. Recently, several patients with vancomycin-resistant $S$. aureus have been identified. Despite the increasing presence of $S$. aureus in the hospital setting, there are only a few studies dealing with the occurrence of $S$. aureus in the student community or potential risk factors for colonization [4]. A recent metaanalysis of some studies showed the prevalence of community-acquired MRSA among hospital patients, as opposed to surveillance cultures conducted at the time of admission, yielded a prevalence of MRSA of $30.2 \%$ [5]. A variety of studies have examined community prevalence of nasal carriage of $S$. aureus in diverse subpopulations, such as adult outpatients, health-care workers (HCWs), college students, and drug abusers [6]. These are issues that may be very useful to clinicians when trying to decide the likelihood that a given patient has a staphylococcal infection, and if so, whether antibiotic coverage should be provided for resistant strains [7]. Exposure to microbes is an inherent risk of working in patient care settings. In view of its increasing incidence in the general population, acquisition of MRSA is a special concern for HCWs [8]. Medical internees comprise a unique population at risk for acquisition of $S$. aureus/MRSA carriage status. The history of continuous exposure to clinical setup during the $4 \frac{1}{2}$ years of course period makes the interns colonize with MRSA. In the first year (pre-clinical), the hospital/patient exposure is very minimal, whereas during the second year (paraclinical), they do get moderate exposure, and in the final year (clinical), the exposure to hospital settings and patients is maximum. The same condition continues even more extensively during the internship for 1 year. The medical course pattern varies from country to country. Hospitalization in the past 12 months and recent skin infection were significantly associated with the risk of being a MRSA carrier [9]. Geriatric male volunteers suffering from chronic sinusitis and not taking antibiotics were at higher risk for carrying $S$. aureus [6]. No study has analyzed whether this patient exposure creates a risk of $S$. aureus carriage and MRSA acquisition for Indian medical students, interns in particular. This study was designed to analyze the nasal carriage status among interns of tertiary care teaching hospital with special emphasis on $S$. aureus/MRSA and their antibiotic sensitivity pattern with any associated risk factors.

\section{METHODS}

This study was conducted in the Clinical Microbiology Laboratory of tertiary care teaching hospital, Mangalore, India, between July and August 2013.

\section{Study design}

This was a type of cross-sectional study. A sample size of 168 was calculated considering power of $80 \%$, confidence level of $95 \%$, and a 
relative precision $6 \%$ and prevalence of nasal carriage of MRSA to be $21 \%$ and non-response rate of $10 \%[6]$.

The formula used was as follows:

$\mathrm{N}=4 \mathrm{pq} / \mathrm{d}^{2}$

Where $\mathrm{p}$ is prevalence, $\mathrm{q}$ is $1-\mathrm{p}$, and then, $\mathrm{d}$ is relative precision.

A battery of 150 internees was randomly recruited, and the study proposal was certified by the Institutional Ethical Committee. The details including age, sex, life style, current skin infection/other illness, and other relevant information about the participants were obtained using designed pro forma. Swabs from both anterior nares were collected with a sterile swab and processed bacteriologically by inoculating onto mannitol salt agar (MSA) and was incubated appropriately. The colonies were identified as $S$. aureus using standard tests including colony morphology, Gram-stain, catalase test, and coagulase test [10]. The isolated strains of $S$. aureus were screened for methicillin resistance by modified Kirby-Bauer method using cefoxitin $(30 \mu \mathrm{g})$ disc on Mueller-Hinton agar (MHA) [11], and the isolates with inhibition zone diameter $\leq 21 \mathrm{~mm}$ around cefoxitin disc were considered as MRSA strains [12]. Screening for vancomycin susceptibility was also done using vancomycin E strip on MHA. Further, antibiotic susceptibility testing for all isolates of $S$. aureus was done using antibiotics such as amoxicillin/clavulanic acid (20/10 $\mu \mathrm{g})$, ciprofloxacin $(5 \mu \mathrm{g})$ ceftriaxone $(30 \mu \mathrm{g})$, cotrimoxazole $(23.75 / 1.25 \mu \mathrm{g})$, erythromycin $(15 \mu \mathrm{g})$, gentamicin $(10 \mu \mathrm{g})$, linezolid $(30 \mu \mathrm{g})$, penicillin (10 units), and teicoplanin $(30 \mu \mathrm{g})$ by the modified Kirby-Bauer method.

Tests were analysed by adjusting the inoculum to 0.5 McFarland standard. S. aureus ATCC 25923, MRSA ATCC 29213, and MSSA ATCC 33591 were used as controls under similar conditions. Antibiotic sensitivity testing and result interpretation were done according to the Clinical and Laboratory Standards guidelines [13]. Repeat samples were collected from all the participants who showed the nasal carriage of MRSA after an interval of 15 days and processed in the same manner as mentioned above for confirmation. The common implications of this present investigation were to know the prevalence of $S$. aureus and MRSA carriage among the internees in a single institution, findings of this study may be utilized in the development of specific health education and/or promotion activities for those who are currently colonized in this population through an institution-wide program on MRSA and detecting the carriers and treating the same, and it is possible to minimize the transmission of MRSA.

\section{Statistical analysis}

Results were be compelled and tabulated and all data were subjected to the Statistical Package for the Social Sciences version 17.0 .The results were presented in the form of tables and graphs. Associations of different factors were done by using CHICSQUARE test and $p$ value $<0.05$ were considered as significant.

\section{RESULTS AND DISCUSSIONS}

Among the 150 internees (age ranging from 22 to 24) were screened and analyzed microbiologically for nasal carriage of S.aureus, 78 (52\%) were males and $72(48 \%)$ were females (Table 1$)$.

The types of organisms isolated from the anterior nares of the subjects were shown in Table 2. Among the isolates, $S$. aureus was found to be predominant 71 (47.3\%) followed by coagulase-negative Staphylococcus 55 (36.7\%). During the study, MSA was found to be very useful for the quick identification of $S$. aureus.

The antibiotic susceptibility pattern of $S$. aureus was shown in Fig. 1 . Out of 150, 71 participants were positive for nasal carriage of S. aureus (47.3\%).
Table 1: Gender wise distribution of participants, carriage status of $S$. aureus and MRSA

\begin{tabular}{llll}
\hline Gender & $\begin{array}{l}\text { Number of } \\
\text { participants (\%) }\end{array}$ & $\begin{array}{l}\text { S. aureus } \\
\text { carriers (\%) }\end{array}$ & MRSA carriers \\
\hline Male & $78(52)$ & $39(54.9)$ & 1 \\
Female & $72(48)$ & $32(45.1)$ & 0 \\
Total & 150 & 71 & 1 \\
\hline
\end{tabular}

MRSA: Methicillin-resistant Staphylococcus aureus, S. aureus: Staphylococcus aureus

Table 2: Nature of organisms isolated from anterior nares

\begin{tabular}{ll}
\hline Organisms isolated & $\mathbf{n}(\mathbf{\%})$ \\
\hline S. aureus & $71(47.3)$ \\
CoNS & $55(36.7)$ \\
No growth & $15(10)$ \\
Diphtheroids & $4(2.7)$ \\
Micrococcus & $3(2)$ \\
Candida & $2(1.3)$ \\
\hline
\end{tabular}

CoNS: Coagulase-negative Staphylococcus, S. aureus: Staphylococcus aureus

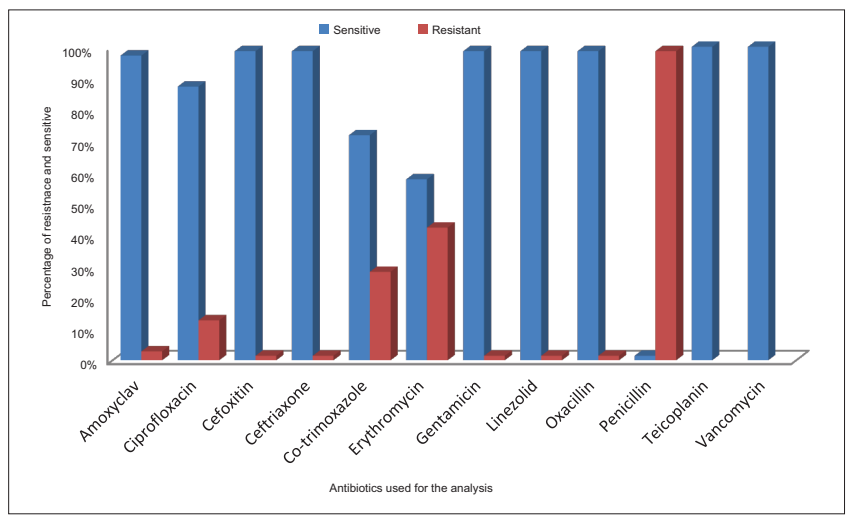

Fig. 1: Antibiotic susceptibility profile of Staphylococcus aureus isolates

Only one among the 71 isolates of S.aureus turned out to be MRSA (1.4\%) (Fig. 2). Overall MRSA nasal carriage rate was $0.67 \%$ in this study. Usage of cefoxitin disc was found to be convenient over oxacillin disc in the determination of MRSA.

Out of the 71 isolates of $S$. aureus, $98.6 \%$ were resistant to penicillin, whereas resistance to erythromycin was $42.3 \%$. Eventually, all $S$. aureus isolates were $100 \%$ susceptible to vancomycin, linezolid, and teicoplanin. The only one MRSA isolate was resistant to all the antibiotics except vancomycin, linezolid, and teicoplanin. In relation to various factors associated with $S$. aureus carriage status, $39 \%$ of the participants were on antibiotics/nasal sprays $(\mathrm{p}=0.121), 31 \%$ were smokers $(p<0.005), 30 \%$ were suffered from allergies $(p=0.43)$, and $11 \%$ had snoring problems ( $\mathrm{p}=0.363$ ) (Fig. 3 ).

\section{DISCUSSION}

The anterior nares of humans found to be the primary ecological niches of the $S$. aureus [14]. The carriage patterns are of three types. Approximately $20 \%$ of the individuals almost always carry one type of strain, and they are called persistent carriers. A large proportion of the population $(60 \%)$ harbors $S$. aureus intermittently, and the strains change with varying frequencies. Such persons are called intermittent carriers. Finally, minorities of the people $(20 \%)$ almost never carry S. aureus, and they are called non-carriers. The reasons for these differences in the colonization patterns are unknown [15]. It is interesting and necessary to detect the $S$. aureus and MRSA nasal carriage rates of a part of HCWs such as internees of medical colleges. 


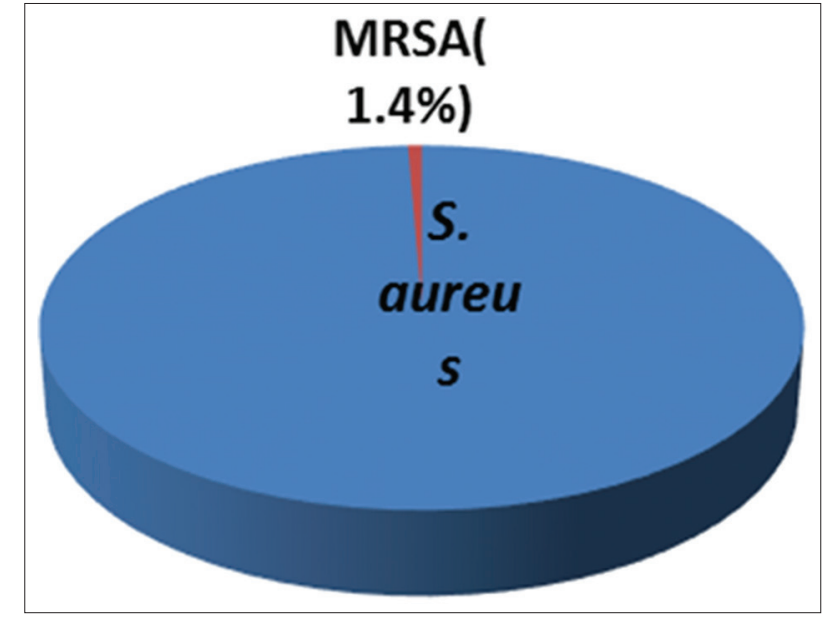

Fig. 2: Percentage of methicillin-resistant Staphylococcus aureus among the $S$. aureus isolates

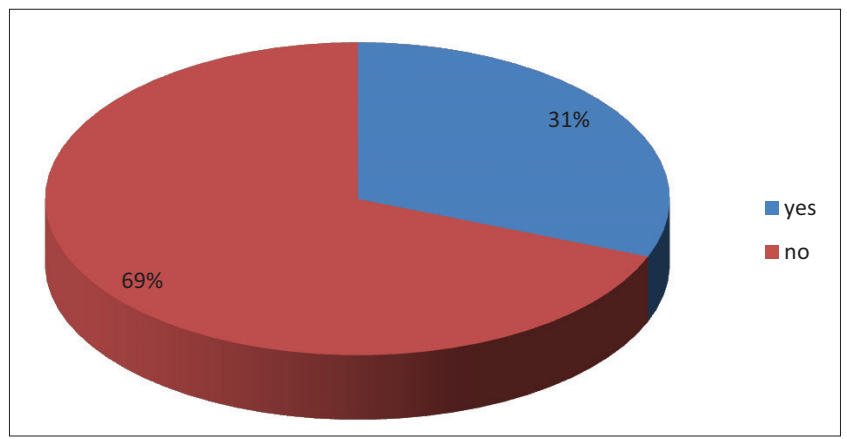

Fig. 3: Relationship between smoking habits and Staphylococcus aureus carriage status

We had 187 internees in 2013-2014 batch, out of which 150 voluntarily participated in the present study representing $80 \%$. It was presumed that these individuals may acquire the carriage status of $S$. aureus/MRSA during their continuous interaction with the patients and thereby may act as a potential source of infection to other patients, resulting in nosocomial infections $[4,9,16]$.

The prevalence of $S$. aureus and MRSA varies between institutions and geographic areas. The differences in the study design such as the sample size and the method employed for S. aureus and MRSA detection may account for the disparity in the carriage rate $[4,17]$. Nasal carriage of $S$. aureus among Internees of Kasturba Medical College, Mangalore, India, according to the result of this study was $47.3 \%$. Out of 71 isolates of $S$. aureus, one was MRSA positive. The $S$. aureus carriage rate of internees recorded in this study was at par with the general population, whereas MRSA carriage rate was significantly low in spite of their continuous exposure with patients in clinical settings [18,19]. A similar kind of study was conducted in our institution last year by involving secondyear MBBS students [20]. It was observed that $S$. aureus carriage rate among the said student population was $52.1 \%$ and the overall carriage rate of MRSA was $6.1 \%$, and among the $S$. aureus carriers, $11.5 \%$ were MRSA. Compared to the above-said study, the $S$. aureus carriage rate among the internees was marginally low (52.1\% and $47.3 \%)$ and MRSA was drastically less $(6.1 \%$ and $0.67 \%)$, respectively. Various studies around the world have evaluated nasal carriage rates of $S$. aureus and MRSA in students of medical profession. One of the studies from India by involving medical students of Malaysian base revealed nasal carriage rate of $23.7 \%$ of $S$. aureus and no MRSA [21]. Another study from Thailand disclosed $29.68 \%$ of $S$. aureus nasal carriage rate and again with zero percentage of MRSA [22]. Whereas the outcome of two more studies from pre-clinical students of Duhok University and Saudi
Arabia were $9.2 \%$ and $18.7 \%$ of $S$. aureus and $4.6 \%$ and $6.7 \%$ of MRSA, respectively $[23,24]$. Medical internees are the population, where they are being continuously exposed to clinical setup from the past 4 years of the MBBS course. Hence, by this study, the answer to the question "Is Acquisition of S. aureus and MRSA an Occupational Hazard for Medical Students?" was "Not necessarily." It was observed that among the various risk factors such as the use of antibiotics, allergies, snoring, and smoking only smoking were found to be statistically significant $(<0.05)$ in nasal carriage status of $S$. aureus.

The repeated isolation of MRSA from the participant who was found to be MRSA carrier in the first time confirmed that he is a persistent MRSA carrier. The only one isolate of MRSA was sensitive to vancomycin, linezolid, and teicoplanin. Both parents of the MRSA carrier reported in this study were found to be in medical profession. Hence, it was not clear that the acquisition of MRSA carriage status was whether due to close contact with the parents or exposure to the patients in the hospital. It is recommended to screen the parents too for MRSA carriage status and if they also found to be MRSA carriers to treat all of them. As MRSA isolated was sensitive to vancomycin, teicoplanin, and linezolid, and it is not difficult to treat the carrier. Aiming at $0 \%$ carriage rate of MRSA among the internees of our institution the identified MRSA carrier may be treated with mupirocin and further screened for carriage status to check the efficacy of treatment. However, periodic screening for MRSA should be an ongoing practice in medical student population. This can be seen as a task because this particular group with the highest frequency of contact with the patients and the most likely to transmit this superbug. Further, advantage of treating the carriers and institution of effective hospital control policies is in prevention of its transmission to their family members and others in the community.

The single most important factor for preventing nosocomial infections is compliance of the health professionals with the sanitary and the antibacterial guidelines. To achieve this, the health professionals should be informed about the potential consequences of the nosocomial infections, both inside and outside the hospital, and their cooperation should be sought to diminish the carriage of $S$. aureus. Simple preventive measures such as hand washing before and after the patient examination, the use of sterile aprons and masks in the post-operative wards, awareness during the examination of immunocompromised patients, and avoiding touching one's nose during work can reduce the disease transmission rate considerably. All the HCWs should be periodically educated and trained about the maintenance of hygiene and infection control and the effects of the use or rather, the misuse of antibiotics.

\section{CONCLUSION}

S. aureus carriage among Internees of Kasturba Medical College, Mangalore, was $47.3 \%$. All the $S$. aureus strains were sensitive to vancomycin, teicoplanin, and linezolid. A large majority of the isolates (98.6\%) were resistant to penicillin. Out of 71 isolates of S. aureus, only one was found to be MRSA (1.4\%). The overall carriage rate of MRSA was $0.67 \%$ in the present study group. The only one MRSA isolate was resistant to all the antibiotics except vancomycin, linezolid, and teicoplanin. In relation to various factors associated with $S$. aureus carriage status, $39 \%$ of the participants were on antibiotics/nasal sprays ( $p=0.121), 31 \%$ were smokers ( $\mathrm{p}<0.005), 30 \%$ were suffered from allergies $(\mathrm{p}=0.43)$, and $11 \%$ had snoring problems $(\mathrm{p}=0.363)$. Only smoking $(\mathrm{p}<0.05)$ was found to be statistically significant as a risk factor associated with the acquisition of $S$. aureus carriage status among the internees. Hence, by this study, the answer to the question "Is Acquisition of S. aureus and MRSA an Occupational Hazard for Medical Students?" was "Not necessarily."

\section{ACKNOWLEDGMENT}

Authors would like to thank the Manipal University for providing facilities for carrying out this research work and the technical staff of Department of Microbiology, Kasturba Medical College, Mangalore. This work was supported by Indian Council of Medical. Research STS 2013 project. 


\section{REFERENCES}

1. Foster TJ. The Staphylococcus aureus 'superbug'. J Clin Invest 2004;114(12):1693-6.

2. Peacock SJ, de Silva I, Lowy FD. What determines nasal carriage of Staphylococcus aureus? Trends Microbiol 2001;9(12):605-10.

3. Deresinski S. Methicillin-resistant Staphylococcus aureus: An evolutionary, epidemiologic, and therapeutic odyssey. Clin Infect Dis 2005;40(4):562-73.

4. Chambers HF. The changing epidemiology of Staphylococcus aureus? Emerg Infect Dis 2001;7(2):178-82.

5. Salgado CD, Farr BM, Calfee DP. Community-acquired methicillinresistant Staphylococcus aureus: A meta-analysis of prevalence and risk factors. Clin Infect Dis 2003;36(2):131-9.

6. Bischoff WE, Wallis ML, Tucker KB, Reboussin BA, Sherertz RJ. Staphylococcus aureus nasal carriage in a student community: Prevalence, clonal relationships, and risk factors. Infect Control Hosp Epidemiol 2004;25(6):485-91.

7. Albrich WC, Harbarth S. Health-care workers: Source, vector, or victim of MRSA? Lancet Infect Dis 2008;8(5):289-301.

8. Eveillard M, Martin Y, Hidri N, Boussougant Y, Joly-Guillou ML. Carriage of methicillin-resistant Staphylococcus aureus among hospital employees: Prevalence, duration, and transmission to households. Infect Control Hosp Epidemiol 2004;25(2):114-20.

9. Rohde RE, Denham R, Brannon A. Methicillin resistant Staphylococcus aureus: Carriage rates and characterization of students in a Texas university. Clin Lab Sci 2009;22(3):176-84

10. Cheesbrough M. District Laboratory Practice in Tropical Countries. Part 2. Cambridge: Cambridge University Press; 2008.

11. Bauer AW, Kirby WM, Sherris JC, Turck M. Antibiotic susceptibility testing by a standardized single disk method. Am J Clin Pathol 1966;45(4):493-6

12. Clinical and Laboratory Standards Institute. Surveillance for MRSA: Principles, Practices, and Challenges; A Report. CLSI Document X07-R. Wayne, PA: Clinical and Laboratory Standards Institute; 2010 .

13. Clinical and Laboratory Standards Institute. NCCLS Performance Standards for Antimicrobial Disc Diffusion Tests; Approved Standards, CLSI Document M2-M9. $9^{\text {th }}$ ed. Wayne, PA: Clinical and Laboratory Standards Institute; 2006.

14. Peacock SJ, Justice A, Griffiths D, de Silva GD, Kantzanou MN, Crook D, et al. Determinants of acquisition and carriage of Staphylococcus aureus in infancy. J Clin Microbiol 2003;41(12):5718-25.

15. Kluytmans J, van Belkum A, Verbrugh H. Nasal carriage of Staphylococcus aureus: Epidemiology, underlying mechanisms, and associated risks. Clin Microbiol Rev 1997;10(3):505-20.

16. Robicsek A, Beaumont JL, Peterson LR. Duration of colonization with methicillin resistant Staphylococcus aureus. Clin Infect Dis 2009;48(7):910-3

17. Bellows C, Smith A, Wheeler J, Morici L. Nasal carriage of methicillinresistant Staphylococcus aureus among students at a Louisiana medical university. Braz J Infect Dis 2013;17(1):118-9.

18. Amiry AA. Methicillin resistant Staphylococcus aureus: An occupational health hazard in the prehospital setting. J Acute Dis 2015;4(7):274-6.

19. Susethira AR, Saiprasanna N, Vigenshwari RS, Uma A, Prabhu N, subramanian TK. Methicillin resistant Staphylococcus aureus-nasal carriage among healthcare workers. J Glob Biosci 2015;4(4):251824.

20. Radhakrishna M, Taneja A, Rao P. Nasal carriage of Staphylococcus aureus with special emphasis on methicillin resistant Staphylococcus aureus among students of a south Indian medical college-prevalence and antibiogram pattern. Asian J Pharm Clin Res 2016;9 Suppl 2:129-32.

21. Santhosh DV, Shobha KL, Bairy I, Rao G, Anand KM, D’Souza J. Nasal screening and survey of pre-clinical medical students from Malaysia for nasal carriage of coagulase positive MRSA and rate of nasal colonization with Staphylococcus species. J Clin Diagn Res 2007;1(6):494-9.

22. Treesirichod A, Hantagool S, Prommalikit O. Nasal carriage and antimicrobial susceptibility of Staphylococcus aureus among medical students at the HRH Princess Maha Chakri Sirindhorn Medical Center, Thailand: A cross sectional study. J Infect Public Health 2013;6(3):196-201.

23. Ma XX, Sun DD, Wang S, Wang ML, Li M, Shang H, et al. Nasal carriage of methicillin-resistant Staphylococcus aureus among preclinical medical students: Epidemiologic and molecular characteristics of methicillin-resistant Staphylococcus aureus clones. Diagn Microbiol Infect Dis 2011;70(1):22-30

24. Zakai SA. Prevalence of methicillin-resistant Staphylococcus aureus nasal colonization among medical students in Jeddah, Saudi Arabia. Saudi Med J 2015;36(7):807-12. 